\title{
Film Formation on Mild Steel and Low Alloy Steels in Aerated Sea Water*
}

\author{
By Toshio SHIBATA, ${ }^{* *}$ Go OKAMOTO, ,* Atsuhiko MURAO, ${ }^{* * *}$ and Takeshi TSUCHIDA ${ }^{* *}$
}

\section{Synopsis}

A simple model to explain the difference of corrosivity of various kinds of steels in sea water is proposed. The model assumes the existence of two series resistances of the diffusion of oxygen. One of the resistances is related to the diffusion layer in the liquid phase and the other is caused by the precipitated film of corrosion products.

The latter resistance changes with the time of immersion and by the addition of alloying elements in steels. The resistance as a whole is determined with the aid of an electrochemical technique. Evaluation of the diffusion constant of oxygen through the film and the volume of the film produced on mild steel indicates that the film has a gel-like structure and its thickness is larger than that of dry oxide owing to hydration or incorporation of anions. The effects of chromium and copper as an alloying element is discussed in view of the change of the diffusion constant of oxygen and the volume of oxide film produced.

\section{Introduction}

Low alloy steels containing a minor element such as copper and chromium have been widely used because of their good resistance to atmospheric corrosion and marine corrosion.

A large number of studies suggest that the rate of corrosion of steel in the atmosphere decreases owing to the formation of protective oxide films. In sea water, however, it is noted that the rate of corrosion does not change with the composition of metals and alloys and it is determined only by the diffusion of dissolved oxygen. ${ }^{1)}$ Nevertheless careful investigations indicate that the rate of corrosion in sea water varies slightly with an addition of alloying element to steels. ${ }^{2}$ )

In this study we proposed a simple model assuming the presence of two kinds of diffusion resistance of oxygen combined in series to explain the difference of corrosivity of various kinds of alloy steels. One of the resistances is caused by the precipitation films formed during corrosion and the other is determined by the liquid diffusion layer adjacent to the film surface.

The reaction resistance of the oxygen reduction was determined by an electrochemical method and the change of the resistance with the amount of alloying of copper and chromium was discussed.

\section{Theory of Estimating the Reaction Resistance of Oxygen Reduction}

When iron is immersed in an aerated neutral solution, the dissolution reaction of iron proceeds spontaneously by coupling the anodic reaction

$$
\mathrm{Fe} \longrightarrow \mathrm{Fe}^{2+}+2 e
$$

and the cathodic reaction

$$
\mathrm{O}_{2}+2 \mathrm{H}_{2} \mathrm{O}+4 e \longrightarrow 4 \mathrm{OH}^{-}
$$

Usually, the rate of dissolution is controlled by the diffusion of oxygen from the solution.

The corrosion rate $\left(i_{\text {eorr }}\right)$ is proportional to the difference between the concentrations of oxygen in the bulk solution $(c)$ and at the metal/solution interface $\left(c^{\prime}\right)$. Thus,

$$
i_{\text {corr }}=4 F k_{2}\left(c-c^{\prime}\right)=i_{d}
$$

where $i_{d}$ is the diffusion current expressed in $\mathrm{A} / \mathrm{cm}^{2}, k_{2}$ the reaction constant in $\mathrm{cm} / \mathrm{sec}, F$ the Faraday constant and 4 the number of electrons required for the reduction of one molecule of oxygen.

If the rate of oxygen reduction at the metal/solution interface is the first order with respect to the concentration of oxygen at the interface, the diffusion current is expressed by

$$
i_{d}=4 F k_{1} c^{\prime}
$$

Elimination of the concentration $\left(c^{\prime}\right)$ from Eqs. (3) and Eq. (4) gives

$$
i_{d}=c /\left(1 / 4 F k_{1}+1 / 4 F k_{2}\right)
$$

or

$$
1 / i_{d}=\left(1 / 4 F k_{1}+1 / 4 F k_{2}\right) 1 / c \ldots
$$

When the rate of oxygen consumption at the interface is sufficiently large, i.e.

$$
k_{1} \gg k_{2}
$$

only the diffusion of oxygen in the solution side determines the corrosion rate and Eq. (5b) is reduced to

$$
1 / i_{d}=1 / 4 F k_{2} c=1 / i_{1 \mathrm{im}}
$$

However, if the rate of oxygen consumption decreases due to a change in property of the interface (in this case the surface is covered with the oxide film), the condition of Eq. (6) does not hold and Eq. (7) should be replaced by Eq. (5b).

The value of $k_{2}$ can be determined from Eq. (7) by measuring $i_{\text {lim }}$ when Eq. (6) is valid. The value of $k_{2}$ can be calculated alternatively by using the following equation which is a well known dimensionless equation of mass transfer :

$$
k_{2} d / D_{2}=A(\mathrm{Re})^{\alpha}(\mathrm{Sc})^{\beta}
$$

In this equation $d$ is the diameter of cylinder, $D_{2}$ the

* Presented at the 74th ISIJ Meeting, October 2, 1967 in Sapporo. Manuscript received October 11, 1968.

** Corrosion Center, Faculty of Engineering, Hokkaido University, Kita Junijo, Sapporo 063.

*** Nippon Kokan K.K., Minami-Watarida, Kawasaki 210. 
diffusion constant of oxygen and $A, \alpha$ and $\beta$ are constant.

By using the value of $k_{2}$ thus obtained and measuring the diffusion current and the concentration of oxygen in the solution, the reaction resistance $1 / k_{1}$ for oxygen reduction at the interface can be determined by the following Eq. (9).

$$
1 / k_{1}=\left(1 / i_{d}-1 / i_{1 \mathrm{im}}\right) \times 1 / 4 F C .
$$

\section{Experiment}

Mild steel, copper steel, chromium steel and CORTEN steel* were used as specimens. Their compositions are shown in Table 1.

Cylindlical electrodes of the diameter of $30 \mathrm{~mm}$ and the height of $28 \mathrm{~mm}$ were machined from ingots of steels and both ends of the cylinder were covered with wax and vinyl chloride tube of equal diameter. The electrode surface was polished with $0 / 4$ emery paper and etched in $20 \% \mathrm{HNO}_{3}$ for $20 \mathrm{sec}$, followed by etching in $10 \% \mathrm{H}_{2} \mathrm{SO}_{4}$ for $1 \mathrm{~min}$ at room temperature before immersion into sea water.

Sea water was artificially prepared in the laboratory to give the composition of $\mathrm{NaCl} 32.237 \mathrm{~g}, \mathrm{MgCl}_{2} 5.76$ and $\mathrm{Na}_{2} \mathrm{SO}_{4} 39.86 \mathrm{~g}$ in one liter. The concentration of oxygen in sea water was determined by the Winkler method.

The electrolytic cell was a cylindrical glass vessel of $1 l$ capacity as shown in Fig. 1 . The volume of the solution used was $900 \mathrm{ml}$. A platinum electrode (f) to supply the current and two gas distributors (b) to dissolve air or nitrogen were provided. The potential of the electrode (a) was measured with a saturated calomel electrode via a bridge (c) filled with the solution. All of the potential data are referred to the saturated calomel electrode. The cylindrical electrode rotating was connected electrically by way of a mercury pool (a) to a potentiostat. The speed of rotation can be changed in the range from 0 to $900 \mathrm{rpm}$, but most of experiments were carried out at $400 \mathrm{rpm}$. Temperature of the cell was kept constant in a water bath at $25^{\circ} \pm 0.01^{\circ} \mathrm{C}$ unless otherwise stated.

Two electrolytic cells of the same size were used for the measurement of current density-potential curves (i.e. polarization curves). In the first experiment the steel electrode was put in one of the cells with or without rotation. The solution was always saturated with air. The polarization curves were obtained at different times of immersion by changing the potential to lower

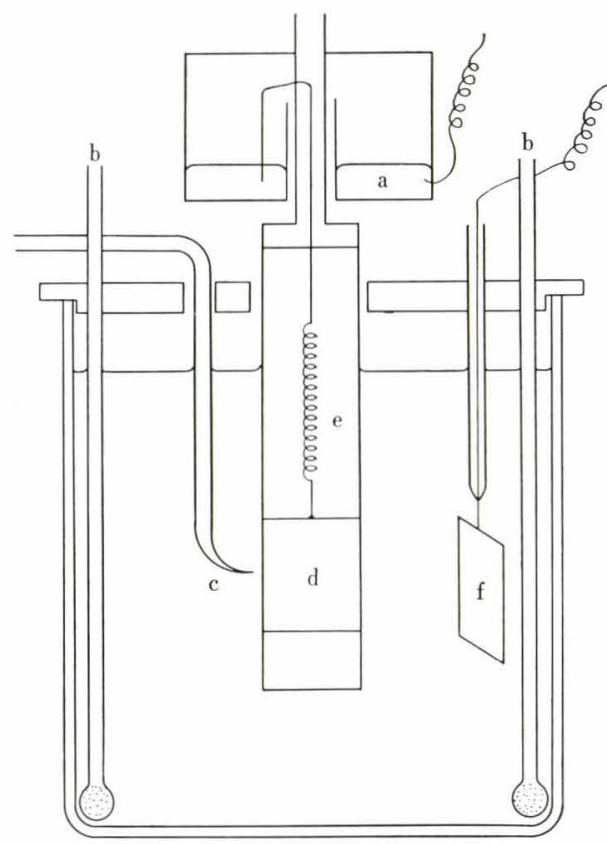
(a) Mercury pool
(b) Gas distributor to dissolve air or nitrogen gas
(c) Tip of capillary to sce
(d) Gylindrical electrode
(e) Holder
(f) Counter Pt electrode

Fig. 1. Electrolytic cell

values at a constant rate of $100 \mathrm{mV} / \mathrm{min}$. The measurement of the curves was always made at the rotation speed of $400 \mathrm{rpm}$, irrespective of the rotation speed applied during the immersion period. In another experiment, the electrode was immersed in aerated sea water for various periods of time as in the case of first experiment and then transferred to the second cell which had been saturated with nitrogen. The polarization curves were obtained in this second cell with the same potential-sweep rate as described above.

\section{Results and Discussion}

\section{Polarization Curves}

A typical example of polarization curves is shown in Fig. 2. This is obtained for CORTEN steel specimen without rotation before the polarization curves was measured at $400 \mathrm{rpm}$. The result shows that the cathodic current decreases with the increase of time of immersion. At the beginning of immersion a middle

Table 1. Composition of steels

\begin{tabular}{l|ccccc}
\hline & $\mathrm{C}$ & $\mathrm{Si}$ & $\mathrm{Mn}$ & $\mathrm{Cr}$ & $\mathrm{Ni}$ \\
\hline $\mathrm{COR}$ TEN & 0.122 & 0.34 & 0.28 & 0.55 & 0.46 \\
$2 \% \mathrm{Cr}$ & 0.127 & 0.33 & 0.39 & 1.92 & 0.38 \\
$3 \% \mathrm{Cr}$ & 0.127 & 0.33 & 0.39 & 0.38 & 0.08 \\
$0.1 \% \mathrm{Cu}$ & 0.08 & 0.36 & 0.39 & 0.21 \\
$0.2 \% \mathrm{Cu}$ & 0.04 & 0.37 & 0.28 & 0.60 \\
$0.6 \% \mathrm{Cu}$ & 0.07 & 0.22 & 0.31 &
\end{tabular}

* The authors are grateful to Fuji Iron \& Steel Co., Ltd. for the gift of those steels. 


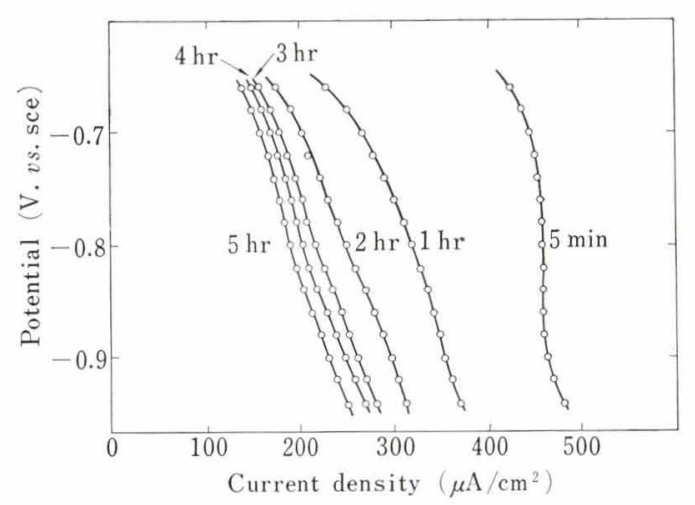

Fig. 2. Cathodic polarization curves measured on the cylindrical electrode rotating at $400 \mathrm{rpm}$ in aerated sea water

part of the curve is almost parallel to the potential axis, that is, a limiting current is observed distinctly. However, this parallel part of the curve becomes to incline as the time elapsed.

Such a change in the slope of the curve may be explained by the contribution of the cathodic reduction of oxide films formed during immersion. In order to see how the reduction of oxide film takes place at the surface, the steel electrode covered with the oxide film was polarized in the solution saturated with nitrogen. The results obtained are shown in Fig. 3 which indicates a considerable reduction of oxide films is occurring. Assuming that the current $\left(i_{\text {obs }}\right)$ observed in the solution saturated with air is the sum of the current of reduction of oxygen $\left(i_{d}\right)$ and the reduction of oxide films $\left(i_{\text {oxide }}\right)$, we get the following relation:

$$
i_{\mathrm{obs}}=i_{\mathrm{oxide}}+i_{d}
$$

Thus $i_{d}$ is obtained as the difference between $i_{\text {obs }}$ and $i_{\text {oxide }}$ (see Figs. 2 and 3 ) for different potentials and periods of immersion. The result is shown in Fig. 4. Since $i_{d}$ vs. potential curves exhibit limiting currents in the potential range from 0.7 to $0.9 \mathrm{~V}$, it can be concluded that the current in this potential region is entirely controlled by the diffusion of oxygen from the solution.

\section{Limiting Current for the Diffusion of Oxygen}

It is found that the reciprocal of the diffusion current changes linearly with the square root of the time of immersion as shown in Fig. 5 . The value of $1 / i_{\text {lim }}$ defined by Eq. (7) can be obtained by extrapolating the linear relation between $1 / i_{d}$ and $\sqrt{t}$ to the time of zero. This is because the electrode surface is not covered with oxide film at the beginning so that the rate of the reaction at the interface is much larger than that of oxygen diffusion (Eq. (6)).

Values of $1 / i_{\text {lim }}$ determined by this extrapolation method are given in the last line of Table 2 for various speeds of rotation.

It is found from Table 2 that values of $1 / i_{\text {lim }}$ decrease with the increase in the speed of rotation. As explained in the preceding section, the value of $k_{2}$ can be obtained by calculation using Eq. (8). In a number of studies concerning to the mass transfer

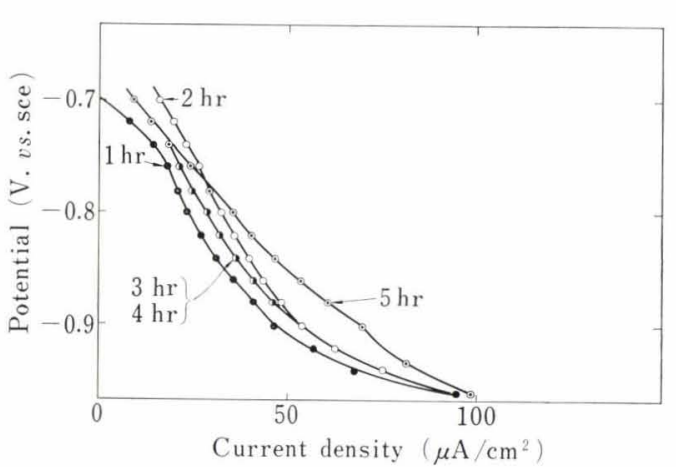

Fig. 3. Cathodic polarization curves measured on the electrode covered with corrosion products in deaerated sea water



Fig. 4. Corrected curves of cathodic polarization

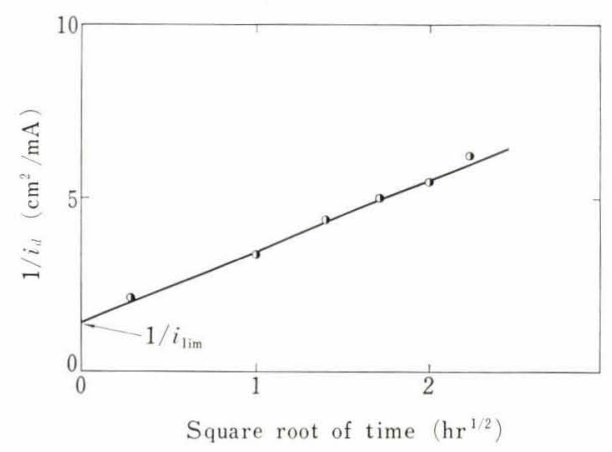

Fig. 5. Reciprocal current density of diffusion as a function of square root of time

Table 2. Values of $1 / i_{1 \mathrm{im}}$ calculated by using Eqs. (7) and $\left(8^{\prime}\right)$ in comparison with measured one at various speeds of rotation

\begin{tabular}{|c|c|c|c|c|}
\hline$\omega(\mathrm{rpm})$ & 200 & 400 & 700 & 920 \\
\hline $\operatorname{Re}\left(\times 10^{4}\right)$ & 0.90 & 1.81 & 3.16 & 4.26 \\
\hline $\mathrm{Re}^{0.62}$ & 284 & 436 & 617 & 742 \\
\hline $\mathrm{k}_{2}\left(\times 10^{-3} \mathrm{~cm} / \mathrm{sec}\right)$ & 0.603 & 0.924 & 1.310 & 1.500 \\
\hline $1 / i_{1 \mathrm{im}} \cdot \operatorname{cal} .\left(\mathrm{cm}^{2} / \mathrm{mA}\right)$ & 2.09 & 1.36 & 0.961 & 0.838 \\
\hline $1 / i_{1 \mathrm{im}}$. meas. $\left(\mathrm{cm}^{2} / \mathrm{mA}\right)$ & 2.36 & 1.40 & 0.90 & 0.80 \\
\hline
\end{tabular}

problems, evaluation of the constants in Eq. (8) have been made and their theoretical background was dis-

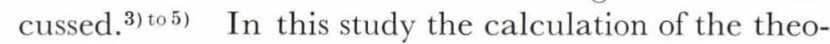
retical values of $k_{2}$ was performed by using the following Eq. (8') which had been given by Okamoto and 
others $^{6)}$ in their studies on the corrosion of mild steel in aerated acid solutions :

$$
k_{2}=0.35(\mathrm{Re})^{0.62}(\mathrm{Sc})^{0.356}\left(D_{2} / d\right) .
$$

Equation (7) is used to estimate the value of $1 / i_{1 \mathrm{im}}$. Calculation was carried out by using the following figures for variables: $d=2.88 \mathrm{~cm}, D_{2}=1.95 \times 10^{-5} \mathrm{~cm}^{2} / \mathrm{sec}$, $\mathrm{C}=2.06 \times 10^{-7} \mathrm{~mol} / \mathrm{cm}^{3}, \rho=1.02 \mathrm{~g} / \mathrm{cm}^{3}, \mu=0.98 \times 10^{-2}$ poise. Values of Reynolds number and Schmidt number are given by $\mathrm{Re}=\mathrm{du} \rho / \mu=d^{2} \rho \pi \omega / 60=45.2 \omega$ and $\mathrm{Sc}=\mu / \rho D_{2}=493$ where $\omega$ is speed of rotation (rpm). Values of $k_{2}$ and $1 / i_{1 \mathrm{~m}}$ calculated are respectively given in the third and the fourth lines of Table 2. Calculated and experimental data for $1 / i_{1 \mathrm{im}}$ are in a good agreement as seen in Table 2 except for the smallest number of Reynolds. This coincidence strongly supports the concept that $i_{1 \mathrm{im}}$ indicated in Fig. 5 is the current corresponding to the diffusion of oxygen from the solution. Therefore, it can be concluded that the increase of $1 / i_{d}$ from $1 / i_{1 \mathrm{im}}$ with time as indicated in Fig. 5 is caused by the change of the resistance of oxygen reduction owing to the film formation during immersion. The change of reaction resistance $1 / k_{1}$ caused by the formation of the film can then be calculated by using the Eq. (9).

\section{Mechanism of Film Growth}

Changes* of the reaction resistance $1 / k_{1}$ for several kinds of alloy steels are plotted against the square root of the time of immersion (Figs. 6 and 7).

Figure 6 shows the increase of the reaction resistance with time for some steels which were immersed in sea water on stagnant condition. The specimens were rotated at $400 \mathrm{rpm}$ when the measurement of polarization curves is carried out. Figure 7 shows the results obtained in the experiments in which steel electrodes were rotated continuously at $400 \mathrm{rpm}$ during immersion and also during the measurement of polarization curves.

The increase of resistance on the stagnant condition is much slower than the one on the rotating condition as can be seen from a comparison of Fig. 6 with Fig. 7. On rotating conditions, because of the resistance of the diffusion of oxygen from the solution is smaller than

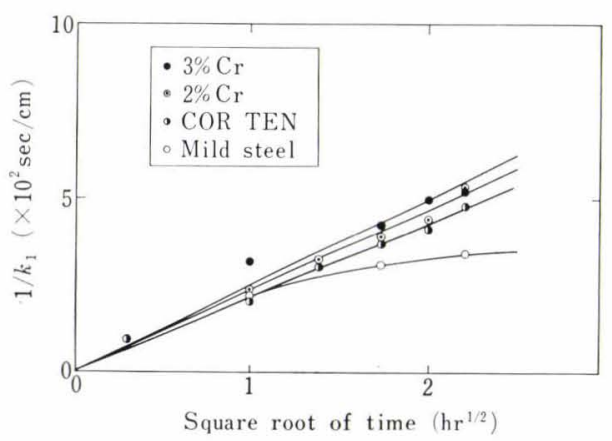

Fig. 6. Increase of reaction resistance with time on the electrodes immersed without rotation

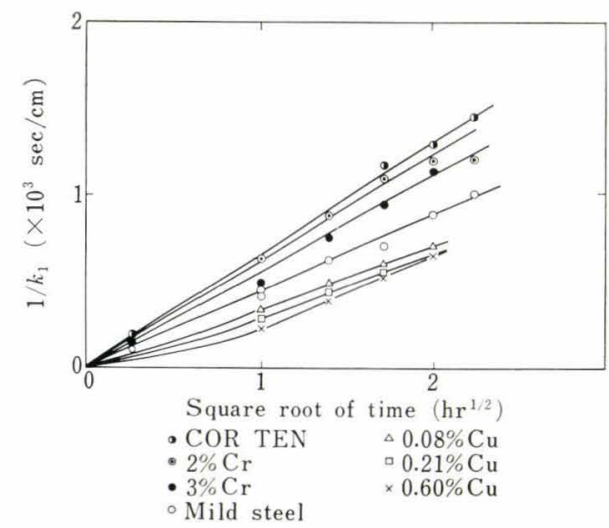

Fig. 7. Increase of reaction resistance with time on the electrodes rotating at $400 \mathrm{rpm}$ during immersion

that on the stagnant condition, (i.e. larger quantity of oxygen is supplied to the surface) so that the film produced at the surface must be thicker than that obtained on the stagnant condition. The growth of the film to such a thickness will lead to the change of the nature of metal/solution interface or the reaction resistance of oxygen reduction at the interface. It is obvious from Figs. 6 and 7 that the reaction resistance for any kind of steel increases linearly with the square root of the time of immersion. The relation observed is related to the rate law of the parabolic growth of the film which is usually observed in the oxidation of metals at high temperatures.

The temperature dependency of the reaction resistance for mild steel is shown in Fig. 8. As can be seen, the higher the rate of increase of the resistance is, the lower the temperature is.

Figure 9 shows the growth of film with time at various temperatures. Film thickness was determined by observations of a sectional area of the electrode by the aid of microscope. It is found that there is also a parabolic relation between the thickness and the time of the immersion, which, however, has an opposite temperature dependency to the one observed for the reaction resistance shown in Fig. 8.

It seems likely that soluble ferrous ions produced by corrosion are changed to ferric ions by further oxidation at the interface of metal/solution. Except for



Fig. 8. Increase of reaction resistance on mild steel at various temperatures

\footnotetext{
* After $4 \mathrm{hr}$ of immersion the change of $1 / k_{1}$ was scarcely observed for all kinds of steels. This fact is consistent with well-known observation that the corrosion rate in sea water does not change appreciably with time. Our mechanism of the reaction of oxygen reduction at the beginning of immersion can not be applied to the understanding of the corrosion process after many hours.
} 


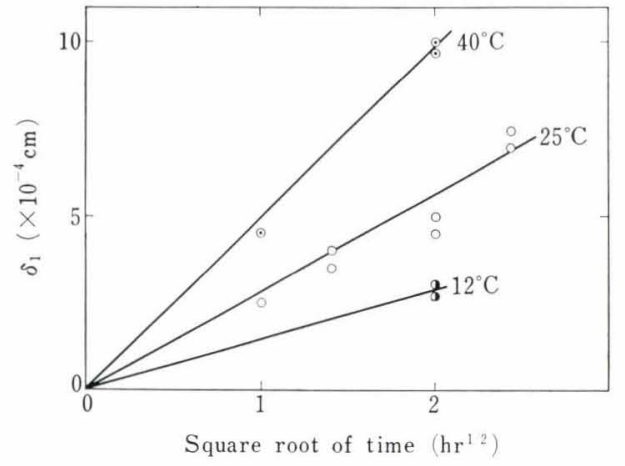

Fig. 9. Growth of film on mild steel with time

those transferred to the bulk solution, ferric ions thus formed will precipitate on the steel surface to form ferric oxide or magnetite films containing water or anions as liegand molecules. Since the ratio of the amounts of the transferred and the precipitated ferric irons is observed to be constant, it is quite certain that the thickness $\left(\delta_{1}\right)$ of the precipitated film is proportional to the amount of iron corroded (or oxygen consumed) $(q)$, thus

$$
\delta_{1}=\alpha q
$$

where $\alpha$ is a proportionality constant corresponding to the volume of oxide produced by one mole of oxygen reacted. Assuming that the growth of the film is determined by the rate of diffusion of oxygen through the film, the resistance $\left(1 / k_{1}\right)$ of oxygen reduction at the surface is given by

$$
1 / k_{1}=\delta_{1} / D_{1}
$$

where $D_{1}$ is the diffusion constant of oxygen in the film.

By using Eqs. (11) and (12) and integrating Eq. (5a) under the assumption that the resistance $\left(1 / k_{2}\right)$ is neglected, we obtain

$$
1 / k_{1}=\sqrt{C \alpha / D_{1}} \sqrt{t}
$$

Rearrangement of Eq. (13) gives the following equation which expresses a relation between $\delta_{1}$ and $\sqrt{t}$

$$
\delta_{1}=\sqrt{C \alpha D_{1}} \sqrt{t}
$$

As can be seen in Fig. 8, $1 / k_{1}$ measured is proportional to $\sqrt{t}$. This is consistent with Eq. (13). The observed relation between $\delta_{1}$ and $\sqrt{t}$ (Fig. 9) is also found to agree with the one predicted by Eq. (14). Consequently, it is clear that $\alpha$ and $D_{1}$ can be determined from the experimental results shown in Figs. 8 and 9 by using Eqs. (13) and (14) together.

Plotting $D_{1}$ thus obtained against reciprocals of absolute temperatures (Fig. 10), we obtain

$$
D_{1}=1.06 \times 10^{8} \exp (-19200 / R T)
$$

The value of $D_{1}$ at $25^{\circ} \mathrm{C}$ is $6.91 \times 10^{-7} \mathrm{~cm}^{2} / \mathrm{sec}$. This is one thirties of the value of $D_{2}\left(=1.95 \times 10^{-5}\right)$, the diffusion constant in the bulk solution. However, $D_{1}$ is much larger than the diffusion constant of oxygen $\left(=1.09 \times 10^{-26}\right)$ in magnetite. The latter value is ob-

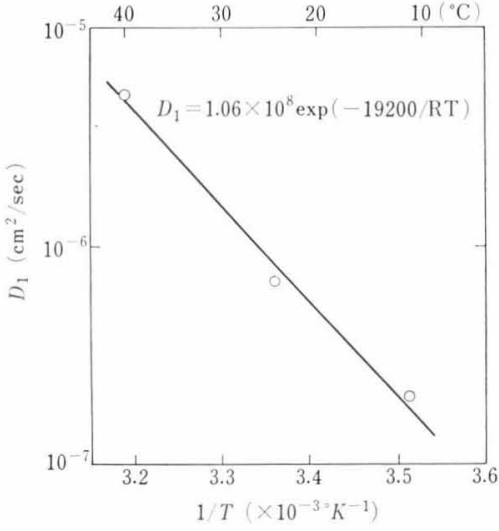

Fig. 10. Diffusion constant of oxygen in the film formed on mild steel against reciprocal of absolute temperature

tained by an extraporation method from the data reported by Castle and Surman ${ }^{7}$ who measured the diffusion constant in magnetite which is placed in water vapour atmosphere in the temperature range between $302^{\circ}$ and $550^{\circ} \mathrm{C}$. It is concluded that the diffusion constant of oxygen through the film is quite different from the one in the bulk oxide and rather similar to the one in the solution.

The value of $\alpha$ calculated from Eqs. (13) and (14) is $52.4 \mathrm{~cm}^{3}$ of oxide per one mole of oxygen. If $Y$ is the amount of precipitated iron divided by that of dissolved one and $X$ is the volume of oxide produced from one mole of oxygen in stoichiometric composition, $\alpha$ is equal to $X Y$. From the chemical analysis of iron in the solution after the corrosion experiment, the value of $Y$ was found to be 0.8 . It gives therefore $X=$ $65.5 \mathrm{~cm}^{3} / \mathrm{mol}$. If we assume that the film is composed of $\mathrm{Fe}_{2} \mathrm{O}_{3}$ whose density is equal to 5.12 , its volume produced from one mole of oxygen is calculated to be $22.3 \mathrm{~cm}^{3} / \mathrm{mol}$. If the film is composed of $\mathrm{Fe}(\mathrm{OH})_{3}$, its volume will be $42.0 \mathrm{~cm}^{3} / \mathrm{mol}$.

It was found that the volume of the film actually precipitated is somewhat larger than these calculated volumes of $\mathrm{Fe}_{2} \mathrm{O}_{3}$ and $\mathrm{Fe}(\mathrm{OH})_{3}$. The film is considered to be thickened by hydration of incorporation of anions and it may have a gel-like structure. This is the reason why the value of diffusion constant in the film is quite similar to the one in the solution as described above.

\section{Effect of Addition of Alloying Element}

An addition of chromium to steel leads to the increase of reaction resistance as can be seen in Figs. 6 and 7. In the case of the stagnant immersion, larger increase of the resistance with time is observed, the more the amount of chromium added.

It was found, however, that the reaction resistance decreases with the increase in chromium content under the rotating condition, though this is not the case for mind steels. Thus, the rate of increase is in the order of CORTEN (0.55 $\mathrm{Cr} \%)>2 \% \quad \mathrm{Cr}>3 \% \quad \mathrm{Cr}>$ mild steel.

The thickness of the film formed on chromium steels was too thin to measure by the microscopic method so that it may be concluded in this case that the contribu- 
tion of $D_{1}$ to the increase of $1 / k_{1}\left(=\delta_{1} / D_{1}\right)$ is considerably larger than that of $\delta_{1}$. In other words, the addition of chromium to the steel inhibits the precipitation of oxide and makes diffusion of oxygen in the film difficult. Thus, the rate of corrosion comes to be small.

The fact that the order of the increase of resistance with the amount of chromium is different for conditions with or without rotation is explained to be caused by the change of precipitation ratio $\alpha$. This change is brought about from an increased rate of transfer of ferric ions to the bulk solution owing to agitation. Such an explanation is quite reasonable because the film produced on the surface is easily detached from the surface by a gentle rubbing with finger.

An addition of copper to steel decreases the reaction resistance as can be seen in Fig. 7. Microscopic observation revealed that the film was thin compared with the one on mild steel. This fact suggests that the addition of copper causes only a slight change of the diffusion constant but retards effectively the precipitation of oxide as films on the surface. Such a retardation $^{8)}$ is suggested in the study of the precipitation and dissolution behaviour of colloidal ferric hydroxide containing copper.

In this study we discussed only the initial process of corrosion within $4 \mathrm{hr}$. After $4 \mathrm{hr}$ the rate of corrosion does not change appreciably with time, though the precipitation or the film growth is still taking place. Further studies on the precipitation of corrosion product and its inhibitive action on corrosion are needed to clarify the corrosion process proceeding spontaneously in sea water.

\section{Summary and Conclusions}

A simple model of explaining the difference of corrosivity of various kinds of alloy steels in sea water is proposed. The model is based on the combination of two resistances of diffusion of oxygen in series. The first resistance is caused by the formation of the solid film of corrosion products and the second by the liquid diffusion layer adjacent to the film surface. The value of resistance is determined with the aid of the electrochemical method which makes use of measuring the diffusion current of oxygen on the steel surface.

The diffusion constant of oxygen through the film on mild steel is quite similar to the one in the bulk solution rather than in the bulk oxides. In addition, the volume of the film produced is found to be larger than that of $\mathrm{Fe}_{2} \mathrm{O}_{3}$ or $\mathrm{Fe}(\mathrm{OH})_{3}$.

These results suggest that the film is thickened by hydration or some incorporation of anions and has a gel-like structure so that oxygen can diffuse quite easily. The addition of chromium to the steel inhibits the precipitation of oxide and makes diffusion of oxygen in the film difficult so that the rate of corrosion is reduced. The addition of copper does not change the diffusion constant appreciably but retards the precipitation of oxide to form films on the surface.

\section{Acknowledgements}

The authors express their appreciation to Dr. N. Sato and Dr. M. Nagayama for their discussions throughout this work.

\section{REFERENCES}

1) H. H. Uhlig: Corrosion and Corrosion Control, (1963), 87, John Wiley \& Sons, Inc.

2) F. L. Laque and H. R. Copson: Corrosion Resistance of Metals and Alloys, 2nd Ed., (1963), 331, Reinhold Pub. Company.

3) H. Kubota and K. Kashiwaya: J. Faculty Eng. Hokkaido Univ., 9 (1953), 148 (in Japanese).

4) W. Brötz: Grundriss der Chemischen Reaktiontechnik, (1958), 135, Verlag Chemie.

5) T. K. Sherwood and R. L. Pigford: Adsorption and Extraction, 2nd Ed., (1952), McGraw-Hill Book Co., Inc.

6) G. Okamoto and H. Kubota: Denki Kagaku (J. Electrochem. Soc. Japan, 22 (1954), 56 (in Japanese).

7) J. E. Castle and P. L. Surman: J. Phys. Chem., 71 (1967), 4255.

8) R. Furuichi and G. Okamoto: Unpublished. 